\title{
Feynman e as Integrais de Trajetória
}

\section{Feynman Path Integrals}

\author{
J. David M. Viana*1,2 \\ ${ }^{1}$ Universidade de Brasília, Instituto de Física, Centro Internacional de Física da Matéria Condensada, Campus Darcy Ribeiro, \\ Brasília, DF, Brasil \\ ${ }^{2}$ Universidade Federal da Bahia, Instituto de Física, Campus Universitário de Ondina, Salvador, BA, Brasil
}

Recebido em 05 de Dezembro, 2017. Revisado em 27 de Janeiro, 2018. Aceito em 15 de Fevereiro, 2018.

O método de integrais de trajetória, desenvolvido por Feynman no artigo "Space-Time Approach to NonRelativistic Quantum Mechanics" de 1948, é uma das formulações da teoria quântica que se junta, considerando a época do artigo, a duas anteriores: (i) a formulação, de certa forma o padrão apresentado em livros-textos, desenvolvida na década de 1920 por Schrödinger, Heisenberg, Dirac, Von Neumann, Born, Jordan e outros, e que se baseia no espaço de Hilbert e operadores que atuam nesse espaço, e (ii) a descrição no espaço de fase, também conhecida como a quantização de Moyal, que se baseia na função quasi-distribuição de Wigner proposta em 1932 e na lei de Correspondência de Weyl de 1927, e conduz à estrutura matemática não comutativa baseada no produto estrela $(\star)$. Neste trabalho, apresentamos uma revisão pedagógica de pontos considerados básicos para o desenvolvimento de Feynman. Destacamos a linha de pesquisa de Dirac na busca de analogias entre a Mecânica Clássica e a Mecânica Quântica, bem como os postulados formulados por Feynman no artigo acima citado, aspectos importantes para a compreensão da teoria mas pouco conhecidos e pouco divulgados de forma completa. Um exemplo é apresentado para elucidar o método e sua relação com a equação de Schrödinger dependente do tempo. Palavras-chave: mecânica quântica, integrais de trajetória, princípio da ação mínima.

The method of path integrals developed by Feynman in the paper "Space-Time Approach to Non-Relativistic Quantum Mechanics" of 1948, is one way of formulating the quantum theory. This development joins previous ones: (i) the formulation developed by Schrödinger, Heisenberg, Dirac, Von Neumann, Born and Jordan, which is based on the Hilbert space, and (ii) the phase space picture also known as Moyal's Quantization which is based on the quasi- distribution function of Wigner proposed in 1932 and leads to the non-commutative mathematical structure defined by the star product $(\star)$. In this work, we present a pedagogical review of points considered basic for the development realised by Feynman. We emphasize Dirac's research line in the search for analogies between the classical and quantum mechanics as well a the postulates formulated by Feynman in the article quoted above. We note that these aspects although important for the comprehension of the path integrals formulation are little known and little divulged of complete form. In order to elucidate the method an example is presented and its relationship to time-dependent Schrödinger equation analyzed.

Keywords: quantum mechanics, path integrals, the action principle.

\section{Introdução}

Atualmente, entre as formas conhecidas de quantizar um sistema microscópico, pode-se destacar três formulações como mais usadas. A primeira, de certa forma o padrão apresentado em livros-textos 1,2], é a baseada no espaço de Hilbert e operadores que atuam nesse espaço 3]; foi desenvolvida por Schrödinger [4], Heisenberg [5], Dirac [6], von Neumann [7], Born e Jordan [8] e outros na década de 1920. A segunda é a formulação no espaço de fase [9], também conhecida como a quantização de Moyal [10]; baseado na função quasi-distribuição de Wigner proposta em 1932 [11, 12] e na lei de correspondência de Weyl de 1927 [13] entre operadores quânticos no espaço de Hilbert e funções no espaço de fase, esse desenvolvimento conduz

*Endereço de correspondência: n1jdavid@gmail.com à estrutura matemática não-comutativa definida pelo produto estrela $(\star) \sqrt{14}-16$. A terceira refere-se à formulação conhecida por integrais de trajetória desenvolvida por Feynman 17]. É essa formulação o motivo do presente artigo. O método de integrais de trajetória de Feynman é reconhecido atualmente como eficiente em várias áreas e, em particular, em teorias de gauge. Desde o surgimento do livro de Feynman e Hibbs [18] vários são os textos que tratam do assunto: alguns em caráter introdutório ou conceitual [19 21]; outros em Teoria de Campos [22, 23] e Mecânica Estatística 24], e ainda outros em aplicações a áreas específicas 25 27], havendo inclusive um Handbook de integrais de trajetória de Feynman [28] onde é possível encontrar uma tabela de integrais de interesse; deve-se também citar a extensão do método de integrais de trajetória para férmions sendo referências importantes 
Berezin 29 e Coleman 30. É usual em vários desses textos, o que também se observa na introdução do artigo "Space-Time Approach to Non-Relativistic Quantum Mechanics" 17], a informação que o desenvolvimento realizado por Feynman tem como origem "uma conjectura de Dirac" ou foi sugerido por "algumas observações de Dirac com respeito à relação da ação clássica e a mecânica quântica". Entretanto, para o melhor de nosso conhecimento, não há uma exposição completa de como surgiram essas sugestões e/ou conjectura de Dirac. Em face desse fato e como forma de preencher essa possível lacuna na literatura, procuramos nesse artigo abordar esse aspecto da formulação do método. $\mathrm{O}$ artigo está dividido em seções: na seção 2 trataremos dos desenvolvimentos de Dirac relativos ao assunto no texto "The Principles of Quantum Mechanics" [6 e no artigo "On the Analogy Between Classical and Quantum Mechanics" [31; na seção 3 para enfatizar a relação do desenvolvimento de Feynman com os aspectos abordados por Dirac, trataremos dos postulados propostos por Feynman [17]; na seção 4 como forma de elucidar a aplicação das ideias de Dirac e Feynman, consideramos um caso específico 18,32 e mostraremos como a amplitude de transição na formulação de Feynman satisfaz a equação de Schrödinger usual. Na seção 2 seguiremos de perto a apresentação de Dirac e, nesse sentido, manteremos sempre que possível sua notação. Assim, para coordenadas, considerando um sistema com $n$ graus de liberdade, temos $q=\left(q_{1}, q_{2}, \ldots, q_{n}\right)$; para os momenta $p=\left(p_{1}, p_{2}, \ldots, p_{n}\right)$; também serão usados $q^{\prime}=\left(q_{1}^{\prime}, q_{2}^{\prime}, \ldots, q_{n}^{\prime}\right), q^{\prime \prime}=\left(q_{1}^{\prime \prime}, q_{2}^{\prime \prime}, \ldots, q_{n}^{\prime \prime}\right) \ldots$ $p^{\prime}=\left(p_{1}^{\prime}, p_{2}^{\prime}, \ldots, p_{n}^{\prime}\right), p^{\prime \prime}=\left(p_{1}^{\prime \prime}, p_{2}^{\prime \prime}, \ldots, p_{n}^{\prime \prime}\right)$ e para os correspondentes bras e kets, $\left\langle q^{\prime}|,| q^{\prime}\right\rangle,\left\langle q^{\prime \prime}|,| q^{\prime \prime}\right\rangle,\left\langle p^{\prime}|,| p^{\prime}\right\rangle$, $\left\langle p^{\prime \prime}|,| p^{\prime \prime}\right\rangle$, respectivamente; as funções quânticas serão notadas por $F(q, p)$ onde $q$ e $p$ são operadores, e as correspondentes funções clássicas serão designadas por $F_{c}(q, p)$ onde $q$ e $p$ serão variáveis clássicas. Na seção 3 seguiremos Feynman e nas seções seguintes a notação acompanhará a convenção usual onde for possível. Neste sentido deve-se observar que Dirac nota a ação referente a um intervalo de tempo por $S\left(t_{i}, t_{i+1}\right)=\int_{t_{i}}^{t_{i+1}} L(t) d t$, enquanto Feynman nota a mesma ação por $S\left(q_{i+1}, q_{i}\right)$, ou seja, $S\left(q_{i+1}, q_{i}\right)=\int_{t_{i}}^{t_{i+1}} L(t) d t$ simbolizando que $q_{i+1}$ refere-se a $q$ no instante $t_{i+1}$, e $q_{i}$ refere-se a $q$ no instante $t_{i}$.

\section{O desenvolvimento de Dirac}

De acordo com alguns autores 19,23, uma das linhas de pesquisa de interesse de Dirac era a elaboração da Mecânica Quântica com base na analogia com a Mecânica Clássica. De fato, consultando o livro "The Principles of Quantum Mechanics" e o artigo "On the Analogy Between Classical and Quantum Mechanics" observa-se, em várias seções, essa busca de analogia entre as duas mecânicas; em particular há duas seções do livro-texto, em que este fato é bem explícito sendo na seção "The action principle" onde Dirac apresenta a possível relação entre o bra-ket $\left\langle q^{\prime}, t \mid q^{\prime \prime}, t_{0}\right\rangle \equiv\left\langle q_{t}^{\prime} \mid q_{t_{0}}^{\prime \prime}\right\rangle$ e o princípio da ação mínima. Para compreender como essa proposta surge iremos, por razões históricas e porque essa discussão não é apresentada nos desenvolvimentos da formulação das integrais de trajetória, seguir Dirac resumindo os aspectos de interesse para o presente artigo.

\subsection{Movimento de pacote de ondas}

Em seu livro-texto, na seção movimento de pacote de ondas, Dirac chega ao resultado expresso por "vemos deste modo como as equações clássicas do movimento são derivadas da teoria quântica como um caso limite." Para chegar a essa conclusão, Dirac considera um sistema dinâmico com operador Hamiltoniano $H\left(q_{r}, p_{r}\right)$ $(r=1,2, \ldots, n)$ tendo um sistema clássico análogo descrito pela Hamiltoniana $H_{c}\left(q_{r}, p_{r}\right)$, função real obtida usando variáveis algébricas para os operadores $q_{r}, p_{r}$ em $H\left(q_{r}, p_{r}\right)$, e fazendo $\hbar \rightarrow 0$ onde aparecer essa constante em $H$. Dirac propõe, então, que a função de onda dependente do tempo na descrição de Schrödinger seja da forma:

$$
\psi(q, t)=A e^{i \frac{S}{\hbar}}
$$

onde $A$ e $S$ são funções reais de $q$ e $t$, e não variam de forma rápida com seus argumentos.

Ao propor a expressão (1) para $\psi(q, t)$, Dirac procura verificar se essa proposta leva a alguma inconsistência e se há interpretações físicas para as funções $A$ e $S$; para isto leva a eq (1) à equação de Schrödinger

$$
i \hbar \frac{\partial}{\partial t} \psi(q, t)=H \psi(q, t)
$$

o que resulta em

$$
i \hbar \frac{\partial A}{\partial t}-A \frac{\partial S}{\partial t}=e^{-i \frac{S}{\hbar}} H\left(q_{r}, p_{r}\right) A e^{i \frac{S}{\hbar}}
$$

e, explorando o fato que $U=e^{i \frac{S}{\hbar}}$ pode ser visto como um operador linear unitário, mostra que as coordenadas $q$ permanecem inalteradas por $U$ e que, para $p_{r}$, tem-se

$$
e^{-i \frac{S}{\hbar}} p_{r} e^{i \frac{S}{\hbar}}=p_{r}+\frac{\partial S}{\partial q_{r}}
$$

e assim

$$
e^{-i \frac{S}{\hbar}} H\left(q_{r}, p_{r}\right) e^{i \frac{S}{\hbar}}=H\left(q_{r}, p_{r}+\frac{\partial S}{\partial q_{r}}\right)
$$

uma vez que as relações algébricas são preservadas por $U$. Em consequência tem-se, de (3), que:

$$
i \hbar \frac{\partial A}{\partial t}-A \frac{\partial S}{\partial t}=H\left(q_{r}, p_{r}+\frac{\partial S}{\partial q_{r}}\right) A
$$

Chegando à eq (4) Dirac considera o limite $\hbar \rightarrow 0$, abandonando os termos em que $\hbar$ aparece, ou seja, em $i \hbar \frac{\partial A}{\partial t}$ e em $p_{r}$ que é o operador $-i \hbar \frac{\partial}{\partial q_{r}}$ aplicado à função de $q_{r}$. Assim, (4) resulta em

$$
-\frac{\partial S}{\partial t}=H_{c}\left(q_{r}, \frac{\partial S}{\partial q_{r}}\right)
$$


que é a equação diferencial para a função $S$, sendo ela assim determinada pela Hamiltoniana clássica $H_{c}$.

A eq (5), conhecida equação de Hamilton-Jacobi da dinâmica clássica, permite que S seja real e mostra que não há inconsistência em admitir a eq (1) como expressão para $\psi(q, t)$.

Para obter a equação diferencial para $A$, após um desenvolvimento relativamente longo [6] obtém-se que:

$$
\frac{\partial A^{2}}{\partial t}=-\sum_{j} \frac{\partial}{\partial q_{j}}\left\{A^{2}\left[\frac{\partial H_{c}\left(q_{j}, p_{j}\right)}{\partial p_{j}}\right]_{p_{j}=\frac{\partial S}{\partial q_{j}}}\right\}
$$

em que a notação []$_{p_{j}=\frac{\partial S}{\partial q_{j}}}$ significa que se deve substituir, na expressão obtida, $p_{j}$ por $\frac{\partial S}{\partial q_{j}}$ de modo que no final se tenha uma função de $q_{j}$ apenas.

Interpretando $A^{2}$, na eq (6), como a densidade de um fluido no ponto $q$ e instante $t$, Dirac mostra que essa equação pode ser analisada como a equação de conservação desse fluido sendo sua velocidade dada por

$$
\frac{d q_{j}}{d t}=\left[\frac{\partial H_{c}\left(q_{r}, p_{r}\right)}{\partial p_{j}}\right]_{p_{j}=\frac{\partial S}{\partial q_{j}}}
$$

e com $p_{j}$ definido como $\frac{\partial S}{\partial q_{j}}$, segue que

$$
\frac{d p_{j}}{d t}=\frac{d}{d t} \frac{\partial S}{\partial q_{j}}=-\frac{\partial H_{c}\left(q_{r}, p_{r}\right)}{\partial q_{j}}
$$

ou seja, as equações (7) e (8) são equações clássicas de movimento na forma Hamiltoniana mostrando que, no limite $\hbar \rightarrow 0$, são obtidas equações clássicas para o sistema quântico descrito por $H\left(q_{r}, p_{r}\right)$.

\subsection{O princípio da ação}

Na seção 2.1 um dos pontos básicos do desenvolvimento é a introdução da eq (1) onde é definida a função $S$ dependente de $q$ e $t$. Na seção sobre o princípio da ação mínima Dirac continua explorando essa função e escreve

$$
\left\langle q^{\prime}, t \mid q^{\prime \prime}, t=0\right\rangle \equiv\left\langle q_{t}^{\prime} \mid q^{\prime \prime}\right\rangle=e^{i \frac{S}{\hbar}}
$$

com

$$
\left\langle q_{t}^{\prime}\right|=\left\langle q^{\prime}\right| T(t)
$$

sendo $T(t)$ o operador que satisfaz a equação:

$$
i \hbar \frac{\partial T}{\partial t}=H(t) T, \quad T(0)=1
$$

A eq. (9) é agora a definição de $S$, função das variáveis $q_{t}^{\prime}, q^{\prime \prime}$ e explicitamente do tempo.

Comparando a função $S$ da eq. (9) com a função $S$ da eq. (1) Dirac observa que a ausência da função $A$ na eq. (9) exige que $S$ agora seja complexa mas com sua parte real igual a $S$ da eq (1), e sua parte imaginária da ordem de $\hbar$. Desta forma, no limite $\hbar \rightarrow 0$, o $S$ da eq. $(9)$ será igual ao $S$ da eq. (1) e, portanto, satisfará a relação, na notação de Dirac,

$$
-\frac{\partial S}{\partial t}=H_{c}\left(q_{r t}^{\prime}, p_{r t}^{\prime}\right)
$$

onde

$$
p_{r t}^{\prime}=\frac{\partial S}{\partial q_{r t}^{\prime}}
$$

e $H_{c}$ é o Hamiltoniano clássico nas variáveis algébricas $q_{r t}^{\prime}, p_{r t}^{\prime}$. Por outro lado, com $q_{t_{0}}=q$, tem-se

$$
\left\langle q_{t_{0}}^{\prime} \mid q^{\prime \prime}\right\rangle=\delta\left(q_{t_{0}}^{\prime}-q^{\prime \prime}\right)
$$

e o quadrado do módulo de $\left\langle q_{t}^{\prime} \mid q^{\prime \prime}\right\rangle$, i.e. $\left|\left\langle q_{t}^{\prime} \mid q^{\prime \prime}\right\rangle\right|^{2}$, é a probabilidade das coordenadas $q$ terem valores $q^{\prime}$ no instante $t>t_{0}$ se elas tiverem, com certeza, os valores $q^{\prime \prime}$ no instante $t_{0}$. Neste contexto Dirac observa que $\left|q_{t_{0}}^{\prime}\right\rangle$ e $\left\langle q_{t}^{\prime \prime}\right|$ podem ser considerados um auto-ket e um auto-bra do operador posição na descrição de Heisenberg e, como em qualquer instante os auto-kets da posição na descrição de Heisenberg constituem um conjunto completo, tem-se naturalmente

$$
\int d q^{\prime \prime}\left|q_{t}^{\prime \prime}\right\rangle\left\langle q_{t}^{\prime \prime}\right|=1
$$

Além disso, $\left\langle q_{t}^{\prime} \mid q^{\prime \prime}\right\rangle \equiv\left\langle q_{t}^{\prime} \mid q_{t_{0}}^{\prime \prime}\right\rangle$ será solução da equação complexo-conjugada de Schrödinger nas variáveis $q_{t_{0}}^{\prime \prime}$, o que implica na relações

$$
\frac{\partial S}{\partial t_{0}}=H_{c}\left(q_{r}^{\prime \prime}, p_{r}^{\prime \prime}\right)
$$

com

$$
p_{r}^{\prime \prime}=-\frac{\partial S}{\partial q_{r}^{\prime \prime}}
$$

E Dirac conclui que a consequência das equações de Hamilton-Jacobi, (12) e 15 , é que a função $S$, sua solução, é a ação da Mecânica Clássica no intervalo $\left[t_{0}, t\right]$, isto é, a integral da Lagrangiana $L$,

$$
S=\int_{t_{0}}^{t} L\left(t^{\prime}\right) d t^{\prime}
$$

ou seja "a função $S$ definida pela eq. (9) é o análogo quântico da função ação clássica e igual a ela no limite $\hbar \rightarrow 0$ ". Para obter o análogo quântico da Lagrangiana clássica ele considera intervalos de tempo infinitesimais colocando $t=t_{0}+\delta t$ e obtém $\left\langle q_{t_{0}+\delta t}^{\prime} \mid q_{t_{0}}^{\prime \prime}\right\rangle$ como o análogo de $\exp \left[i L\left(t_{0}\right) \frac{\delta t}{\hbar}\right]$, o que segue da eq. (9).

Na sequência, Dirac procura determinar a que corresponde na teoria quântica o princípio da ação mínima e propõe a relação

$$
\exp \left\{\frac{i}{\hbar} \int_{t_{0}}^{t} L\left(t^{\prime}\right) d t^{\prime}\right\}=\exp \left\{i \frac{S\left(t, t_{0}\right)}{\hbar}\right\}=B\left(t, t_{0}\right)
$$

onde $B\left(t, t_{0}\right)$ corresponde a $\left\langle q_{t}^{\prime} \mid q_{t_{0}}^{\prime}\right\rangle$ na teoria quântica. Daí, supondo que o intervalo $\left[t_{0}, t\right]$ seja dividido em um 
grande número de pequenos intervalos de tempo $t_{0} \rightarrow t_{1}$, $t_{1} \rightarrow t_{2}, \ldots, t_{m-1} \rightarrow t_{m}, t_{m} \rightarrow t$, escreve para $B\left(t, t_{0}\right)$

$$
B\left(t, t_{0}\right)=B\left(t, t_{m}\right) B\left(t_{m}, t_{m-1}\right) \ldots B\left(t_{2}, t_{1}\right) B\left(t_{1}, t_{0}\right)
$$

que afirma, pelas propriedades conhecidas dos vetores base $\left|q_{m}^{\prime}\right\rangle$ (vide eq. (13)), corresponde a equação quântica

$$
\begin{aligned}
\left\langle q_{t}^{\prime} \mid q_{0}^{\prime}\right\rangle & =\iint \cdots \int\left\langle q_{t}^{\prime} \mid q_{m}^{\prime}\right\rangle d q_{m}^{\prime}\left\langle q_{m}^{\prime} \mid q_{m-1}^{\prime}\right\rangle d q_{m-1}^{\prime} \\
& \times \cdots\left\langle q_{2}^{\prime} \mid q_{1}^{\prime}\right\rangle d q_{1}^{\prime}\left\langle q_{1}^{\prime} \mid q_{0}^{\prime}\right\rangle
\end{aligned}
$$

onde, para simplificar a notação, $q_{k}^{\prime}$ significa $q_{t k}^{\prime}$.

Dirac faz então uma análise das equações (18) e (19) realçando alguns pontos: (i) que se deve olhar cada fator em (18) como uma função dos $q^{\prime} s$ nos dois pontos extremos do intervalo de tempo a que ele se refere; (ii) que, em consequência, o lado direito da equação (18) é uma função não somente de $q_{t}^{\prime}$ e $q_{t_{0}}^{\prime}$ mas também de todos os $q^{\prime} s$ nos instantes intermediários; (iii) a equação (18) é válida para os valores de $q$ efetivos (trajetória real) nos instantes intermediários; (iv) pequenas variações em torno dos valores efetivos, deixando $S$ estacionária, pela equação (17) também deixarão $B\left(t, t_{0}\right)$ estacionário; (v) é o processo de substituição em (18), dos valores de $q$ nos instantes intermediários, que corresponde em 19 à integração em $q$ naqueles instantes. E conclui que: "O análogo quântico do princípio da ação encontra-se na lei de composição dada em (19)".

O tema de obter uma descrição quântica próxima à descrição clássica, em que as coordenadas $q$ de um sistema dinâmico tenham valores definidos em qualquer instante $t$, é retomado por Dirac ao discutir o conceito de probabilidade no artigo "On the Analogy Between Classical and Quantum Mechanics". Nesse artigo Dirac considera uma sequência de instantes $t_{1}, t_{2}, t_{3}, \ldots$, tão próximos quanto possível um do outro, e trata de como descrever formalmente a probabilidade considerando os valores de $q$, determinados em cada instante e pertencentes em cada instante a pequenos intervalos espaciais; explica então que, desta forma, "terá uma probabilidade formal para a trajetória do sistema, em mecânica quântica, permanecer dentro de certos limites". Especificamente, Dirac considera três instantes $t_{1}, t_{2}, t_{3}$ e que, em cada instante $t_{i}$, haja uma representação cujos vetores básicos sejam $\left|q_{i}^{\prime}\right\rangle$, $\left\langle q_{i}^{\prime}\right|(i=1,2,3)$. Sendo $f\left(q_{1}, q_{2}, q_{3}\right)$ uma função geral das coordenadas nesses instantes e $|\chi\rangle$ um estado arbitrário, ele mostra que:

$$
\begin{aligned}
\left\langle\chi\left|f\left(q_{1}, q_{2}, q_{3}\right)\right| \chi\right\rangle & =\iiint f\left(q_{1}^{\prime}, q_{2}^{\prime}, q_{3}^{\prime}\right)\left\langle\chi \mid q_{1}^{\prime}\right\rangle d q_{1}^{\prime} \\
& \times\left\langle q_{1}^{\prime} \mid q_{2}^{\prime}\right\rangle d q_{2}^{\prime}\left\langle q_{2}^{\prime} \mid q_{3}^{\prime}\right\rangle d q_{3}^{\prime}\left\langle q_{3}^{\prime} \mid \chi\right\rangle
\end{aligned}
$$

onde $q_{i}^{\prime}$ são os valores de $q$ no instante $t_{i}$, e a integração é na região onde os valores de $q_{i}$ na trajetória podem variar.

Observa-se da exposição de Dirac que as bases para o desenvolvimento de uma formulação quântica em termos de trajetórias estão delineadas na equação 20 que se refere diretamente a trajetórias, e nas equações (17), (18) e 19) que buscam uma correspondência com a ação clássica. Há necessidade, no entanto, de um detalhamento para completar tal formulação, o que foi realizado por Feynman em seu artigo "Space-Time Approach to NonRelativistic Quantum Mechanics" 17.

\section{As Integrais de Trajetória de Feynman}

Nesta seção apresentaremos os dois postulados elaborados por Feynman como base da sua formulação e que de certa forma resumem os desenvolvimentos da seção anterior.

\subsection{O primeiro postulado}

Feynman inicia o artigo "Space-Time Approach to NonRelativistic Quantum Mechanics" [17] situando o trabalho como consequência de observações de Dirac sobre a relação da ação clássica com a mecânica quântica. Estabelece então a busca de uma amplitude de probabilidade associada a trajetórias no espaço-tempo em lugar da amplitude de probabilidade associada à posição da partícula em um particular instante de tempo. Com esse objetivo, baseado nos trabalhos que apresentamos na seção 2 Feynman discute, por simplicidade, o caso unidimensional de uma partícula cuja posição $q$ pode assumir vários valores e supõe que tenha sido realizada uma quantidade enorme de medidas sucessivas separadas por pequenos intervalos de tempo $\varepsilon$ : as medidas da coordenada $q$ nos instantes $t_{1}, t_{2}, \ldots, t_{i}, t_{i+1}, \ldots$ são notadas por $q_{1}, q_{2}, \ldots, q_{i}, q_{i+1} \ldots$, respectivamente, o que, com $\varepsilon=t_{i+1}-t_{i}$ define, do ponto de vista clássico, uma trajetória $q(t)$ no limite $\varepsilon \rightarrow 0$. Na sequência Feynman analisa resumidamente o processo de medida comparando as teorias clássica e quântica e introduz o que denomina "medida ideal"; com essa denominação caracteriza, em linhas gerais, a situação em que não é realizada uma medida detalhada da trajetória no sentido de determinar efetivamente cada ponto mas é possível afirmar que a trajetória encontra-se em uma região $R$ do espaço-tempo. Complementando, Feynman afirma que a probabilidade que a partícula seja encontrada por uma "medida ideal" na região $R$ é o quadrado de um número complexo $|\varphi(R)|^{2}$; o número $\varphi(R)$ é denominado amplitude de probabilidade para a região $R$ e dado por

$$
\varphi(R)=\lim _{\varepsilon \rightarrow 0} \int_{R} \Phi\left(\ldots, q_{i}, q_{i+1}, \ldots\right) \ldots d q_{i} d q_{i+1} \ldots
$$

onde $\Phi\left(\ldots, q_{i}, q_{i+1}, \ldots\right)$ é uma função das variáveis $q_{i}$ definindo a trajetória. Em consequência, ele formula o que denomina de primeiro postulado da teoria: "Se uma medida ideal é realizada para determinar se uma partícula tem uma trajetória em uma região $R$ do espaço-tempo, então a probabilidade que o resultado seja positivo é o 
módulo ao quadrado de uma soma de contribuições, uma de cada trajetória na região".

Após esse postulado, Feynman tece considerações sobre o significado dos termos "uma de cada trajetória" e explica: (i) que uma trajetória é definida pelas posições $q_{i}$ determinadas na sequência de intervalos de tempo $\varepsilon=t_{i+1}-t_{i}$ e que todos os valores das coordenadas na região $R$ têm peso igual; (ii) que o valor desse peso depende de $\varepsilon$ e pode ser escolhido de forma que a probabilidade de um evento ocorrer com certeza seja a unidade; (iii) o limite $\varepsilon \rightarrow 0$ deve ser considerado no final dos cálculos.

Deve-se observar na equação 21) que, no limite $\varepsilon \rightarrow 0$, a quantidade $\Phi$ depende da trajetória como um todo sendo natural considerá-la um funcional das trajetórias $q(t)$.

\subsection{O segundo postulado}

Como explica Feynman, enquanto o primeiro postulado estabelece o esquema matemático exigido pela teoria quântica para o cálculo de probabilidades, o segundo postulado trata de calcular a importante quantidade $\Phi$ para cada trajetória. Diz esse postulado: "As trajetórias contribuem igualmente mas a fase de suas contribuições é a ação clássica $S[q(t)]$ (em unidade de $\hbar$ ) i.e., a integral no tempo da Lagrangiana ao longo da trajetória." E Feynman explica que a contribuição $\Phi[q(t)]$ de uma dada trajetória $q(t)$ é proporcional a $\exp \left\{\frac{i}{\hbar} S[q(t)]\right\}$, com

$$
S[q(t)]=\int L(q(t), \dot{q}(t)) d t,
$$

onde a Lagrangiana $L(q(t), \dot{q}(t))$ pode ser uma função explícita do tempo.

Comparando os dois postulados, Feynman aponta que para o primeiro postulado é necessário definir uma trajetória dando apenas a sucessão de pontos $q_{i}$ pelos quais a trajetória passa em instantes sucessivos $t_{i}$. Já para calcular $S[q(t)]$, que aparece no segundo postulado, é necessário conhecer a trajetória em todos os pontos e não apenas em $q_{i}$ o que conduz a admitir que a função $q(t)$ é a trajetória seguida por uma partícula clássica com Lagrangiana $L$ e que, partindo de $q_{i}$ em $t_{i}$, chega a $q_{i+1}$ em $t_{i+1}$. Esta hipótese, afirma Feynman, permite aplicar o segundo postulado a trajetórias descontínuas; o ponto observado é que, apesar das mudanças abruptas de velocidade nos instantes $t_{i}$, não há dificuldade no cálculo da ação já que $L$ depende no máximo da primeira derivada de $q(t)$; como a trajetória clássica é aquela que torna a ação mínima, pode-se escrever:

$$
S=\sum_{i} S\left(q_{i+1}, q_{i}\right)
$$

onde

$$
S\left(q_{i+1}, q_{i}\right)=\min \int_{t_{i}}^{t_{i+1}} L(q(t), \dot{q}(t)) d t
$$

o que exige da mecânica clássica somente o conhecimento da Lagrangiana. Feynman também observa que, na expressão (23), mesmo para $\varepsilon$ finito a soma é infinita por causa da extensão infinita do tempo; daí, para que os postulados tenham significado, deve-se restringir o intervalo de tempo a um valor finito embora suficientemente grande.

Combinando os dois postulados e a relação 23 podese escrever para 21)

$$
\varphi(R)=\lim _{\varepsilon \rightarrow 0} \int_{R} \exp \left[\frac{i}{\hbar} \sum_{i} S\left(q_{i+1}, q_{i}\right)\right] \ldots \frac{d q_{i+1}}{A} \frac{d q_{i}}{A} \ldots
$$

onde o fator de normalização está dividido em fatores $\frac{1}{A}$ para cada instante de tempo e a integração é sobre os valores $q_{i}, q_{i+1}, \ldots$ que estão na região $R$.

Concluindo a seção, Feynman afirma que a equação (25), a definição (24) de $S\left(q_{i+1}, q_{i}\right)$ e a interpretação física de $|\varphi(R)|^{2}$ completam a nova formulação da mecânica quântica. A generalização para um sistema com $k$ graus de liberdade é imediata: neste caso te$\operatorname{mos} q=\left(q^{(1)}, q^{(2)}, \ldots, q^{(k)}\right)$ e uma trajetória será uma sequência de configurações, em instantes sucessivos, descrita no instante $t_{i}$ por $q_{i}=\left(q_{i}^{(1)}, q_{i}^{(2)}, \ldots, q_{i}^{(k)}\right)$, ou seja, o valor de cada uma das coordenadas $q^{(k)}$ em um dado instante $t_{i}$. O símbolo $d q_{i}$ significa neste caso o elemento de volume no espaço de configurações no instante $t_{i}$.

Comentando o conteúdo desta seção, apontaríamos que os postulados enunciados por Feynman constituem na realidade uma síntese e sistematização das ideias desenvolvidas por Dirac em suas exposições a respeito da possível analogia entre a teoria clássica e a teoria quântica; neste contexto, um dos feitos de Feynman é indicar como realizar o cálculo completo de 25 o que elucidaremos na seção 4 e no Apêndice.

Devemos também observar que Feynman em seu artigo, após apresentar os postulados, considerou com sua formulação o conceito de função de onda, a equação de Schrödinger, o limite clássico e outros aspectos como a álgebra de operadores, equação de Newton e uma possível generalização, itens que não abordaremos no presente artigo por estarem além do proposto.

\section{Aplicação}

Para melhor acompanhar o desenvolvimento adotaremos uma notação de bras e kets explicitando posição e tempo, ou seja, em lugar de $\left\langle q_{t}^{\prime \prime} \mid q_{t_{0}}^{\prime}\right\rangle$ escreveremos $\left\langle q^{\prime \prime}, t^{\prime \prime} \mid q^{\prime}, t_{0}\right\rangle$, por exemplo, e para a relação de completeza dos autokets da posição, em lugar da equação (13), usaremos:

$$
\int d q^{\prime \prime}\left|q^{\prime \prime}, t^{\prime \prime}\right\rangle\left\langle q^{\prime \prime}, t^{\prime \prime}\right|=1
$$

Com esta notação, apliquemos a formulação das integrais de trajetória para calcular a amplitude de transição para um sistema (uma partícula) no espaço-tempo ir do 
ponto inicial $\left(q_{1}, t_{1}\right)$ ao ponto final $\left(q_{N}, t_{N}\right)$, considerados fixos.

De acordo com o exposto nas seções anteriores devemos dividir o intervalo $t_{1}-t_{N}$ em $(N-1)$ partes iguais a $\varepsilon$, dadas por:

$$
\varepsilon=t_{j+1}-t_{j}=\frac{t_{N}-t_{1}}{N-1}
$$

e explorando a propriedade de completeza dos auto-kets, segue que

$$
\begin{aligned}
\left\langle q_{N}, t_{N} \mid q_{1}, t_{1}\right\rangle & =\int \cdots \int\left\langle q_{N}, t_{N} \mid q_{N-1}, t_{N-1}\right\rangle d q_{N-1} \\
& \times\left\langle q_{N-1}, t_{N-1} \mid q_{N-2}, t_{N-2}\right\rangle d q_{N-2} \\
& \times\left\langle q_{N-2}, t_{N-2}|\ldots| q_{2}, t_{2}\right\rangle d q_{2} \\
& \times\left\langle q_{2}, t_{2} \mid q_{1}, t_{1}\right\rangle
\end{aligned}
$$

Para o intervalo de tempo $t_{j}-t_{j+1}(j=1,2, \ldots)$ é preciso determinar a amplitude de probabilidade $\left\langle q_{j+1}, t_{j+1} \mid q_{j}, t_{j}\right\rangle$ e, pela expressão (28), integrar em $q_{2}, q_{3}, \ldots, q_{j}, \ldots, q_{N-1}$ o que corresponde a considerar todas as trajetórias no plano $(q, t)$ com os extremos $\left(q_{1}, t_{1}\right)$ e $\left(q_{N}, t_{N}\right)$ fixos, já que $d q_{i}$ varia no instante $t_{i}$ entre os limites que definem a região $R$ onde a partícula pode ser encontrada.

Nota-se assim que, para determinar a amplitude de transição $\left\langle q_{N}, t_{N} \mid q_{1}, t_{1}\right\rangle$ faz-se necessário considerar todas as possíveis trajetórias, ou seja, mesmo aquelas que não têm qualquer semelhança com a trajetória clássica. Como Dirac apontou ao analisar o princípio da ação mínima, isto constitui um aspecto fundamental na diferença entre as duas mecânicas uma vez que na mecânica clássica há uma trajetória definida no plano $(q, t)$ que é obtida considerando a condição de extremo

$$
\delta \int_{t_{1}}^{t_{N}} d t L(q, \dot{q})=0 .
$$

Ainda, de acordo com Dirac (vide seção 3.2), para $\varepsilon=$ $t_{j+1}-t_{j}$

$$
\exp \left\{\frac{i}{\hbar} S\left(t_{j}, t_{j-1}\right)\right\}=B\left(t_{j}, t_{j-1}\right)
$$

corresponde a $\left\langle q_{j} \mid q_{j-1}\right\rangle \operatorname{com} S\left(t_{j}, t_{j-1}\right)=\int_{t_{j-1}}^{t_{j}} L\left(t^{\prime}\right) d t^{\prime}$. Segue, portanto, de (28) que a contribuição de uma trajetória definida será

$$
\begin{aligned}
\prod_{j=2}^{N} \exp \left[\frac{i}{\hbar} S\left(t_{j}, t_{j-1}\right)\right] & =\exp \left[\frac{i}{\hbar} \sum_{j=2}^{N} S\left(t_{j}, t_{j-1}\right)\right] \\
& =\exp \left[\frac{i}{\hbar} S\left(t_{N}, t_{1}\right)\right]
\end{aligned}
$$

ou, na notação de Feynman:

$$
\begin{aligned}
\prod_{j=2}^{N} \exp \left[\frac{i}{\hbar} S\left(q_{j}, q_{j-1}\right)\right] & =\exp \left[\frac{i}{\hbar} \sum_{j=2}^{N} S\left(q_{j}, q_{j-1}\right)\right] \\
& =\exp \left[\frac{i}{\hbar} S\left(q_{N}, q_{1}\right)\right]
\end{aligned}
$$

Agora, para determinar $\left\langle q_{N}, t_{N} \mid q_{1}, t_{1}\right\rangle$, pelas seções 2 e 3 deve-se considerar todas as trajetórias e, pelo segundo postulado de Feynman, tem-se:

$$
\begin{aligned}
\left\langle q_{N}, t_{N} \mid q_{1}, t_{1}\right\rangle & =\lim _{\varepsilon \rightarrow 0} \int \frac{d q_{N-1}}{A} \int \frac{d q_{N-2}}{A} \cdots \\
& \times \int \frac{d q_{2}}{A} \exp \left[\frac{i}{\hbar} \sum_{j=2}^{N} S\left(q_{j}, q_{j-1}\right)\right]
\end{aligned}
$$

ou seja:

$$
\left\langle q_{j}, t_{j} \mid q_{j-1}, t_{j-1}\right\rangle=\frac{1}{A(\varepsilon)} \exp \left[\frac{i}{\hbar} S\left(q_{j}, q_{j-1}\right)\right]
$$

com

$$
S\left(q_{j}, q_{j-1}\right)=\int_{t_{j-1}}^{t_{j}} L(q(t), \dot{q}(t)) d t
$$

com $L$ a Lagrangiana clássica e o fator de normalização dependente somente do intervalo de tempo $\varepsilon=t_{j}-t_{j-1}$ e não do potencial.

Para elucidar a aplicação de (31), (32) e a determinação de $A(\varepsilon)$, seja o caso de uma partícula cuja Lagrangiana é $L(q, \dot{q})=\frac{m \dot{q}^{2}}{2}-V(q)$.

Considerando o intervalo de tempo $\varepsilon$ bem pequeno, pode-se tomar a trajetória entre dois instantes sucessivos como uma linha reta o que, da expressão

$$
S\left(q_{j}, q_{j-1}\right)=\int_{t_{j-1}}^{t_{j}} d t\left[\frac{m \dot{q}^{2}}{2}-V(q)\right],
$$

escrevendo $\dot{q}=\frac{q_{j}-q_{j-1}}{\varepsilon}$, resulta em

$$
\begin{aligned}
S\left(q_{j}, q_{j-1}\right) & =\int_{t_{j-1}}^{t_{j}} d t \frac{m}{2}\left(\frac{q_{j}-q_{j-1}}{\varepsilon}\right)^{2}-\int_{t_{j-1}}^{t_{j}} d t V(q) \\
& =\varepsilon\left\{\frac{m}{2}\left(\frac{q_{j}-q_{j-1}}{\varepsilon}\right)^{2}-V\left(\frac{q_{j}+q_{j-1}}{2}\right)\right\}
\end{aligned}
$$

Em particular, para o caso de uma partícula livre, $V(q)=$ 0 e tem-se, de 31 .

$$
\begin{aligned}
\left\langle q_{j}, t_{j} \mid q_{j-1}, t_{j-1}\right\rangle & =\frac{1}{A(\varepsilon)} \exp \left[\frac{i \varepsilon m}{2 \hbar}\left(\frac{q_{j}-q_{j-1}}{\varepsilon}\right)^{2}\right] \\
& =\frac{1}{A(\varepsilon)} \exp \left[\frac{i m\left(q_{j}-q_{j-1}\right)^{2}}{2 \hbar \varepsilon}\right]
\end{aligned}
$$

o que possibilita determinar o fator $A(\varepsilon)$; de fato, como o mesmo independe do potencial e os kets satisfazem a relação:

$$
\left.\left\langle q_{j}, t_{j} \mid q_{j-1}, t_{j-1}\right\rangle\right|_{t_{j}=t_{j-1}}=\delta\left(q_{j}-q_{j-1}\right),
$$

usando para a função $\delta$ a expressão:

$$
\delta\left(q-q^{\prime}\right)=\lim _{\Delta \rightarrow 0} \frac{1}{\left(\pi \Delta^{2}\right)^{\frac{1}{2}}} \exp \left[-\frac{\left(q-q^{\prime}\right)^{2}}{\Delta^{2}}\right]
$$


obtém-se, de (33), (34) e (35), que:

$$
\frac{1}{A(\varepsilon)}=\sqrt{\frac{m}{2 \pi i \hbar \varepsilon}} .
$$

Assim, para $\varepsilon \rightarrow 0$, segue pelo postulado de Feynman que

$$
\left\langle q_{j}, t_{j} \mid q_{j-1}, t_{j-1}\right\rangle=\sqrt{\frac{m}{2 \pi i \hbar \varepsilon}} \exp \left[\frac{i}{\hbar} S\left(q_{j}, q_{j-1}\right)\right]
$$

e, para a amplitude de transição $\left\langle q_{N}, t_{N} \mid q_{1}, t_{1}\right\rangle$, com $t_{N}-t_{1}$, finito,

$$
\begin{aligned}
\left\langle q_{N}, t_{N} \mid q_{1}, t_{1}\right\rangle & =\lim _{\varepsilon \rightarrow 0}\left(\frac{1}{A(\varepsilon)}\right)^{(N-1)} \int d q_{N-1} \int d q_{N-2} \cdots \\
& \times \int d q_{2} \prod_{j=2}^{N} \exp \left[\frac{i}{\hbar} S\left(q_{j}, q_{j-1}\right)\right]
\end{aligned}
$$

$\operatorname{com} \frac{1}{A(\varepsilon)}=\sqrt{\frac{m}{2 \pi i \hbar \varepsilon}}$, e os pontos do espaço-tempo $\left(q_{N}, t_{N}\right)$ e $\left(q_{1}, t_{1}\right)$ fixos.

A expressão (37) é usualmente escrita como:

$$
\begin{aligned}
\left\langle q_{N}, t_{N} \mid q_{1}, t_{1}\right\rangle & =\int_{q_{1}}^{q_{N}} \mathcal{D}[q(t)] \exp \left\{\frac{i}{\hbar} S[q(t)]\right\} \\
& =\int_{q_{1}}^{q_{N}} \mathcal{D}[q(t)] \exp \left\{\frac{i}{\hbar} \int_{t_{1}}^{t_{N}} L_{c}(q, \dot{q}) d t\right\}
\end{aligned}
$$

e denominada Integral de Trajetória de Feynman. Na equação (38) tem-se a expressão:

$$
\begin{aligned}
\int_{q_{1}}^{q_{N}} \mathcal{D}[q(t)] & =\lim _{\varepsilon \rightarrow 0}\left(\frac{m}{2 \pi i \hbar \varepsilon}\right)^{\frac{(N-1)}{2}} \int d q_{N-1} \\
& \times \int d q_{N-2} \cdots \int d q_{2}
\end{aligned}
$$

definindo uma nova medida no sentido matemático e significando integrar sobre todas as trajetórias indo do ponto $\left(q_{1}, t_{1}\right)$ ao ponto $\left(q_{N}, t_{N}\right)$ fixos, $t_{N}-t_{1}$ finito.

Um fato a observar é que, de $\left\langle q^{\prime \prime}, t \mid q^{\prime}, t_{0}\right\rangle$ com $\left|q^{\prime}, t_{0}\right\rangle$ e $\left\langle q^{\prime \prime}, t\right|$ auto-ket e auto-bra do operador posição na descrição de Heisenberg, tem-se $\left\langle q^{\prime \prime}, t \mid q^{\prime}, t_{0}\right\rangle=$ $\left\langle q^{\prime \prime}\left|e^{-\frac{i}{\hbar} H t} e^{\frac{i}{\hbar} H t_{0}}\right| q^{\prime}\right\rangle=\left\langle q^{\prime \prime}\left|e^{-\frac{i}{\hbar} H\left(t-t_{0}\right)}\right| q^{\prime}\right\rangle$ que é uma das expressões do propagador $\mathcal{K}\left(q^{\prime \prime}, t ; q^{\prime}, t_{0}\right)$. Assim alguns autores 21] ao discutir o método de Feynman dão ênfase ao cálculo do propagador e suas propriedades. No presente volume da RBEF, o conceito de propagador aparece nos trabalhos de Pleitez e Aguilar.

\subsection{A formulação de Feynman e a equação de Schrödinger}

Para analisar a equivalência da formulação de Feynman com a formulação de Schrödinger, busca-se mostrar que a expansão de Feynman para $\left\langle q_{N}, t_{N} \mid q_{1}, t_{1}\right\rangle$ satisfaz a equação de Schrödinger dependente do tempo com relação às variáveis $q_{N}$ e $t_{N}$.
Com esse objetivo é usual considerar a relação

$$
\begin{aligned}
\left\langle q_{N}, t_{N} \mid q_{1}, t_{1}\right\rangle & =\int\left\langle q_{N}, t_{N} \mid q_{N-1}, t_{N-1}\right\rangle d q_{N-1} \\
& \times\left\langle q_{N-1}, t_{N-1} \mid q_{1}, t_{1}\right\rangle
\end{aligned}
$$

que, com a Lagrangiana $L(q, \dot{q})=\frac{m \dot{q}^{2}}{2}-V(q)$ e a expressão de Feynman (36) para o bra-ket $\left\langle q_{N}, t_{N} \mid q_{N-1}, t_{N-1}\right\rangle$, nos dá:

$$
\begin{aligned}
\left\langle q_{N}, t_{N} \mid q_{1}, t_{1}\right\rangle & =\int_{-\infty}^{+\infty} d q_{N-1} \sqrt{\frac{m}{2 \pi i \hbar \varepsilon}} \\
& \times \exp \left[\left(\frac{i m}{2 \hbar}\right) \frac{\left(q_{N}-q_{N-1}\right)^{2}}{\varepsilon}-\frac{i \varepsilon V}{\hbar}\right] \\
& \times\left\langle q_{N-1}, t_{N-1} \mid q_{1}, t_{1}\right\rangle
\end{aligned}
$$

Fazendo a mudança de variável $\eta=q_{N}-q_{N-1} \mathrm{e}$ supondo $q_{N} \rightarrow q$ e $t_{N} \rightarrow t+\varepsilon$, segue que

$$
\begin{aligned}
\left\langle q, t+\varepsilon \mid q_{1}, t_{1}\right\rangle & =\sqrt{\frac{m}{2 \pi i \hbar \varepsilon}} \int_{-\infty}^{+\infty} d \eta \\
& \times \exp \left(\frac{i m \eta^{2}}{2 \hbar \varepsilon}-\frac{i \varepsilon}{m} V\right)\left\langle q-\eta, t \mid q_{1}, t_{1}\right\rangle
\end{aligned}
$$

O fator $\exp \left(\frac{i m \eta^{2}}{2 \hbar \varepsilon}\right)$ oscila de forma muito rápida com $\eta$ uma vez que $\varepsilon$ é infinitesimal e $\hbar$ é uma constante pequena. Quando uma função que oscila rapidamente multiplica uma função bem comportada como o braket $\left\langle q-\eta, t \mid q_{1}, t_{1}\right\rangle$ a integral tende a se anular devido à variação aleatória da fase da exponencial. Daí a contribuição efetiva vem da região onde a fase é estacionária; no presente caso é o ponto $\eta=0$, sendo justificável expandir $\left\langle q-\eta, t \mid q_{1}, t_{1}\right\rangle$ em potências de $\eta$. Também é justificável expandir $\left\langle q, t+\varepsilon \mid q_{1}, t_{1}\right\rangle$ e $\exp \left(-\frac{i \varepsilon V}{m}\right)$ em potências de $\varepsilon$, o que resulta em:

$$
\begin{aligned}
\left\langle q, t \mid q_{1}, t_{1}\right\rangle & +\varepsilon \frac{\partial}{\partial t}\left\langle q, t \mid q_{1}, t_{1}\right\rangle=\sqrt{\frac{m}{2 \pi i \hbar \varepsilon}} \int_{-\infty}^{+\infty} d \eta \\
& \times \exp \left(\frac{i m \eta^{2}}{2 \hbar \varepsilon}\right)\left(1-i \frac{V \varepsilon}{\hbar}+\ldots\right) \\
& \times\left[\left\langle q, t \mid q_{1}, t_{1}\right\rangle+\frac{\eta^{2}}{2} \frac{\partial^{2}}{\partial q^{2}}\left\langle q, t \mid q_{1}, t_{1}\right\rangle+\ldots\right]
\end{aligned}
$$

já que o termo linear $\eta$ é nulo quando integrado. Por outro lado

$$
\int_{-\infty}^{+\infty} d \eta \exp \left(\frac{i m \eta^{2}}{2 \hbar \varepsilon}\right)=\sqrt{\frac{2 \pi i \hbar \varepsilon}{m}}
$$

$\mathrm{e}$

$$
\int_{-\infty}^{+\infty} d \eta \eta^{2} \exp \left(\frac{i m \eta^{2}}{2 \hbar \varepsilon}\right)=\sqrt{2 \pi}\left(\frac{i \hbar \varepsilon}{m}\right)^{\frac{3}{2}}
$$


o que nos dá, substituindo esses valores na relação 41 e mantendo os termos lineares em $\varepsilon$, a equação

$$
\begin{aligned}
\varepsilon \frac{\partial}{\partial t}\left\langle q, t \mid q_{1}, t_{1}\right\rangle & =\sqrt{\frac{m}{2 \pi i \hbar \varepsilon}}(\sqrt{2 \pi})\left(\frac{i \hbar \varepsilon}{m}\right)^{\frac{3}{2}} \\
& \times \frac{1}{2} \frac{\partial^{2}}{\partial q^{2}}\left\langle q, t \mid q_{1}, t_{1}\right\rangle \\
& -\left(\frac{i}{\hbar}\right) \varepsilon V\left\langle q, t \mid q_{1}, t_{1}\right\rangle
\end{aligned}
$$

ou ainda

$$
\begin{aligned}
i \hbar \frac{\partial}{\partial t}\left\langle q, t \mid q_{1}, t_{1}\right\rangle & =-\left(\frac{\hbar^{2}}{2 m}\right) \frac{\partial^{2}}{\partial q^{2}}\left\langle q, t \mid q_{1}, t_{1}\right\rangle \\
& +V\left\langle q, t \mid q_{1}, t_{1}\right\rangle
\end{aligned}
$$

que é a equação de Schrödinger dependente do tempo.

Concluindo essa seção frisaríamos, com as ref. 21, 23, 27, quatro pontos: (i) a definição do fator de normalização $A(\varepsilon)$ não é um problema fácil e não se sabe como resolvê-lo em termos gerais. O fator que aparece na equação (36) normaliza a amplitude de transição, tem um valor prático e com esse valor o limite da equação (37) existe; (ii) a soma sobre todas as trajetórias, necessária para determinar a amplitude de transição $\varphi(b, a)$ entre dois pontos espaço-temporais $\left(a, t_{a}\right)$ e $\left(b, t_{b}\right)$, é outro conceito que ainda merece análise cuidadosa do ponto de vista matemático. Na realidade há diferentes formas de se definir um subconjunto de trajetórias entre dois pontos fixos; a definição a ser usada deve ser a melhor dentro de certos propósitos matemáticos. Muitos textos usam uma notação geral escrevendo como indicado na equação 38

$$
\varphi(b, a)=\int_{a}^{b} \exp \left\{\frac{i}{\hbar} S[b, a]\right\} \mathcal{D}[q(t)]
$$

com $\mathcal{D}[q(t)]$ representando a "medida de integração" adequada ou, como dizem alguns autores, o desconhecimento da natureza precisa da "medida de integração". Trabalhos sobre a medida de integração podem ser encontrados nas ref. [21 26]; (iii) a integral de ação, base de todo o desenvolvimento, aparece nas duas mecânicas mas de forma diferente: na mecânica clássica o que interessa é a forma da integral de ação $S=\int_{t_{a}}^{t_{b}} L(q, \dot{q}, t) d t$ e sua variação. Já na teoria quântica, tanto a forma da integral quanto o valor de seu extremo são de interesse; (iv) um aspecto importante é como são compostos eventos em sucessão. Para compreender essa composição, deve-se lembrar que, se entre $t_{a}$ e $t_{b}$ houver um instante intermediário $t_{c}$, a ação ao longo de qualquer trajetória entre $a$ e $b$ é dada por

$$
S[b, a]=S[b, c]+S[c, a]
$$

e em consequência teremos para a amplitude de transição

$$
\varphi(b, a)=\int \exp \left\{\frac{i}{\hbar} S[b, c]+\frac{i}{\hbar} S[c, b]\right\} \mathcal{D}[q(t)],
$$

expressão que exprime o fato que é possível considerar uma trajetória de $a$ a $b$ dividida em duas partes, i.e. de $q_{a}$ a $q_{c}$ e de $q_{c}$ a $q_{b}$, sendo possível somar sobre todas as trajetórias de $a$ para $c$ e de $c$ para $b$. Tem-se então, após efetuada a soma sobre todas as trajetórias de $a$ a $c$,

$$
\varphi(b, a)=\int_{q_{c}} \int_{c}^{b} \exp \left\{\frac{i}{\hbar} S[b, c]\right\} \varphi(c, a) \mathcal{D}[q(t)] d q_{c}
$$

e somando sobre as trajetórias de $c$ a $b$,

$$
\varphi(b, a)=\int_{q_{c}} \varphi(b, c) \varphi(c, a) d q_{c}
$$

ou seja, as amplitudes para os eventos que ocorrem em sucessão se multiplicam de acordo com a eq. 42.

\section{Considerações finais}

Neste trabalho, visando preencher uma possível lacuna na literatura, apresentamos uma breve revisão sobre pontos considerados básicos, para o desenvolvimento realizado por Feynman, do método de integrais de trajetória. Introduzimos com visão pedagógica o estudo de Dirac sobre analogias entre as mecânicas clássica e quântica. A relação entre o desenvolvimento de Dirac e os postulados apresentados por Feynman é então apontada. Através de um exemplo conhecido e simples, procuramos elucidar o método das integrais de trajetória e sua relação com a equação de Schrödinger dependente do tempo. No Apêndice procuramos resumir os passos a seguir na aplicação do método das integrais de trajetória.

\section{Agradecimentos}

O autor agradece a Dra M. Graças R. Martins pela leitura crítica e sugestões, a Dr. Marco C. B. Fernandes pelas sugestões e indicação de referências, a Dr. Vicente Pleitez por comentário, ao Pedro D. Matos Pereira pela digitação e aos editores Silvio R. A. Salins e Nelson Studart pelo convite para participar deste número da RBEF comemorativo do centenário de R. P. Feynman.

\section{Material Suplementar}

O seguinte material suplementar está disponível online: Apêndice

\section{Referências}

[1] A. Messiah, Quantum Mechanics (North-Holland Publis Comp, Amsterdan, 1973).

[2] L. Landau e E. Lifchitz, Mécanique Quantique (Editions MIR, Moscou, 1966).

[3] R. P. Feynman, Science 152, 699 (1966).

[4] E. Schrödinger, Collected Papers of Wave Mechanics (AMS Chelsea Publis, Providence, Island, 1982).

[5] W. Heisenberg, The Physical Principles of the Quantum Theory (Dover, New York, 1950). 
[6] P.A.M. Dirac The Principles of Quantum Mechanics (Oxford University Press, New York, 1947).

[7] J. von Neumann, Mathematical Foundations of Quantum Mechanics (Princeton University Press, New Jersey, 1955).

[8] W.A. Fedak and J.J. Prentis, Am. J. Phys. 77, 129 (2004).

[9] Y-S Kim and M.E. Noz, Phase Space Picture of Quantum Mechanics: Group Theoretical Approach (World Scientific, Singapore, 1991).

[10] J.E. Moyal Math. Proc. Comb. Philos. Soc. 45, 99 (1949).

[11] E.P. Wigner, Phys. Rev. 40, 749 (1932).

[12] R.G.G. Amorim, M.C.B. Fernandes, A.R. Queiroz, A.E. Santana and J.D.M. Vianna, Rev. Bras. Ens. Fis. 35, 3604 (2013).

[13] H. Weyl, The Theory of Groups and Quantum Mechanics (Dover, New York, 1931)

[14] M.D. Oliveira, M.C.B. Fernandes, F.C. Khanna, A.E. Santana and J.D.M. Vianna, Annals of Physics 312, 492 (2004).

[15] T. Curtright, D. Fairlie and C. Zachos, Phys. Rev. D 58, 025002 (1998).

[16] P. Campos, M.G.R. Martins and J.D.M. Vianna, Phys. Lett. A 381, 1129 (2017).

[17] R.P. Feynman, Rev. Mod. Phys. 20, 367 (1948).

[18] R.P. Feynman and A. Hibbs, Quantum Mechanics and Path Integrals (McGraw-Hill, New York, 1965).

[19] J. Zinn-Justin, Path Integrals in Quantum Mechanics (Oxford University Pres, London, 2004).

[20] G. Roepstorff, Path Integral Approach to Quantum Physics (Springer-Verlag, Berlim, 1996).

21] H.M. Nussenzweig, Integrais de Trajetória Curso Ministrado na I Escola de Verão de Partículas e Campos (1981)

[22] U. Mosel, Path Integrals in Field Theory: An Introduction (Springer-Verlag, Berlim, 2004).

[23] A.F.Rodrigues, A Integral de Feynman in: VIII Escola do CBPF (2010).

[24] A. Wipf, Path Integrals in Quantum and Statistical Mechanics (Springer-Verlag, Berlim, 2013).

[25] H. Kleinert, Path Integrals in Quantum Mechanics, Statistics, Polymer Physics and Financial Markets (World Scientific, Singapore, 2006).

[26] L.S. Schuliman, Techniques and Applications of Path Integrals (John Wiley \& Sons, New York, 1981).

[27] K.C. Mundim, Métodos de Integrais de Trajetória e Sistemas Pseudo-Clássicos Não Relativísticos. Dissertação de Mestrado, UnB, 1982.

[28] C. Grosche and F. Steiner, Handbook of Feynman Path Integrals (John Wiley \& Sons, New York, 1981).

[29] F.A. Berezin, The Method of Second Quantization (Academic Press, New York, 1966).

[30] S. Coleman, The Uses of Instantons in: International School of Subnuclear Physics "Ettore Majorana" (1977).

[31] P.A.M. Dirac, On the Analogy Between Classical and Quantum Mechanics, Rev. Mod. Phys. 17, 195 (1945).

[32] J.J. Sakurai, Modern Quantum Mechanics (AddisonWesley Publis Comp, New York, 1994). 